\title{
STATIC AND DYNAMICS OF A PUMP IMPELLER WITH A BALANCING DEVICE PART II: DYNAMIC ANALYSIS
}

\author{
V.A. MARTSINKOVSKY and A. ZHULYOV \\ Department of General Mechanics and Machine Dynamics \\ Sumy State University \\ R.-Korsakova St., 2, 40007 Sumy, UKRAINE \\ E-mail: marts@omdm.sumdu.edu.ua; alexey.zhulyov@gmail.com \\ C. KUNDERA* \\ Faculty of Mechatronics and Machine Design \\ Kielce University of Technology \\ A1. 1000-lecia PP 7, 25-314 Kielce, POLAND \\ E-mail: kundera@tu.kielce.pl
}

\begin{abstract}
This paper presents the theoretical study of the system comprising an impeller and a balancing device. It deals with the dynamic analysis of the system, i.e., the axial vibrations of the impeller, and the system stability. The dynamic analysis took into account linearized hydrodynamic forces and moments generated in the longitudinal clearances of the seals of the impeller. The theoretical analysis was supplemented with a numerical example with characteristics determined for a real single-stage centrifugal pump.
\end{abstract}

Key words: dynamic analysis, pump impeller, balance device, stability condition.

\section{Introduction}

In impeller pumps, there are systems responsible for balancing or reducing the longitudinal forces acting along the axis of the shaft. Their role is vital as they ensure high durability and reliability of the pump and low energy consumption. While designing a balancing device, it is crucial to enhance the positioning effect of the rotating system and, at the same time, reduce the pump size (Childs, 1993; Jędral, 2001; Korczak, 2005).

The hydrostatic forces as well as inertial, damping, gyroscopic and circular forces and moments generated in the clearances of the balancing device are known to contribute to the changes in the natural and critical frequencies of the impeller and a loss in its dynamic stability, as described, for example, in Refs. (Childs, 1993; Martsinkovsky and Kundera, 2008; Gosiewski, 2008).

In the latest theoretical works (Li at al., 2011; Faria and Miranda, 2012), the transverse vibrations of the impeller are analyzed using a non-linear model of dynamic fluid forces generated in the seals, proposed by Muszynska. The results of the numerical calculations based on the complex non-linear model were represented in the form of dynamic trajectories of the impeller centre, Poincare maps, and bifurcation diagrams.

Dynamic analysis based on linearized models of the impeller-clearance seals system can also be of importance as it enables us to obtain analytical relationships useful in engineering practice.

In their previous work (Kundera and Martsinkovsky, 2014), the authors discuss a single-stage pump with an impeller directly connected to a system of balancing rings. The work describes the design of the balancing device and analyzes statically the whole rotating system.

\footnotetext{
* To whom correspondence should be addressed
} 
This paper is a follow-up theoretical study of the system comprising an impeller and a balancing device. It deals with the dynamic analysis of the system, i.e., the axial vibrations of the impeller, and the system stability. The dynamic analysis takes into account linearized hydrodynamic forces and moments generated in the longitudinal clearances of the seals of the impeller. The results are analytical relationships that can be easily used as the first approximation at the design stage of new impeller-based systems.

\section{Dynamic analysis}

The impeller with a balancing device shown in Fig.1 has three degrees of freedom. It can perform radial, angular and axial vibrations simultaneously.

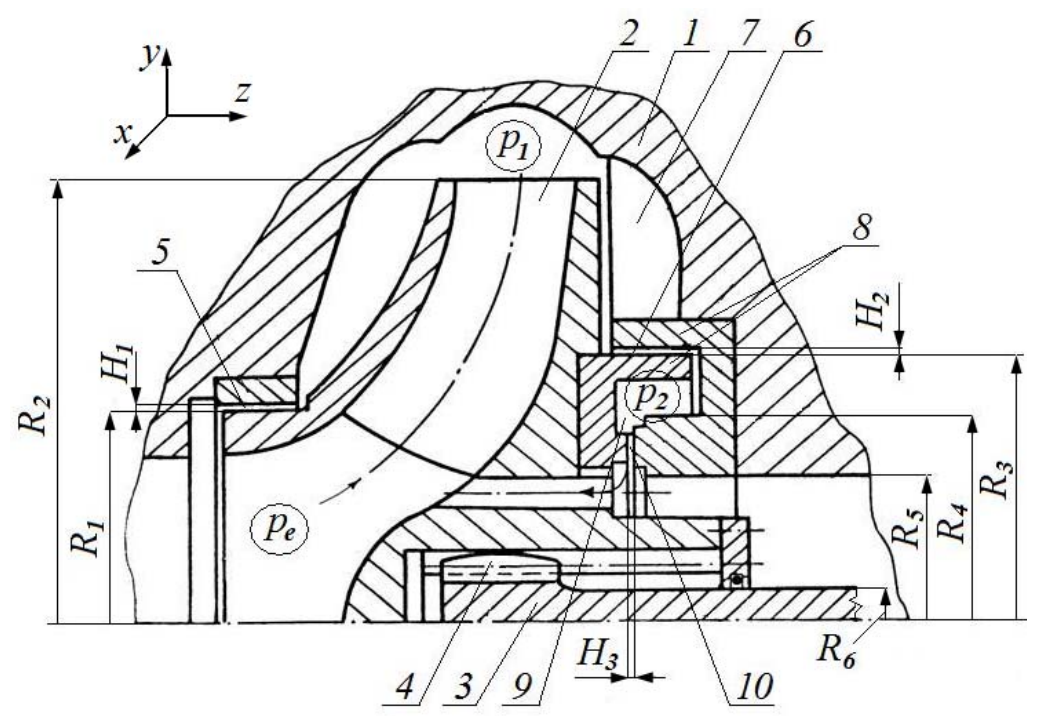

Fig.1. Geometry of the impeller with a balancing device: 1- pump casing, 2- impeller, 3- shaft; 4 - ball joint; 5, 6- longitudinal seals of the impeller; 7 - radial vanes; 8- liner disks of balance device; 9 annular chamber; 10 - lateral (face) clearance.

The axial and radial vibrations of the impeller are due to the eccentricity of the longitudinal clearances and the convergence of the lateral clearance. The correlations described in Ref. (Martsinkovsky and Kundera) are relatively poor for this case because the hydrodynamic moments are one order smaller than the hydrodynamic forces and the influence of the eccentricity of the longitudinal clearances on their capacity is proportional to the value $0.19 \varepsilon^{2}$ (relative eccentricity $\varepsilon=e / H_{1}<1$ ). The shaft deflections (angular vibrations) have even less influence on the capacity of the clearances and, accordingly, on the pressure in chamber 9 (Fig.1) and axial vibrations. Because of the poor correlation between the parameters, in the first approximation, it is possible to analyze the axial vibrations caused by a kinematic excitation in the form of predefined transverse vibrations.

\subsection{Equation of axial vibrations} written as

The equation of the impeller axial vibrations, taking into account the external pressure forces, can be 


$$
m \ddot{z}+c \dot{z}=F_{z}=A_{2} p_{2}+p_{A^{*}} A_{A}-p_{B^{*}} A_{B}-A_{1} p_{1}-A_{e} p_{e} .
$$

After dividing the components in Eq.(2.1) by the product $A_{A} p_{n}$, we obtain the following dimensionless equation of the impeller axial vibrations

$$
T_{1}^{2} \ddot{u}+2 \varsigma T_{1} \dot{u}=\bar{A}_{2} \psi_{2}-\bar{A}_{1} \psi_{1}-\bar{A}_{e} \psi_{e}-\left(K_{1}-K_{2}\right) \Omega^{2}
$$

where parameters and dimensionless quantities

$$
\begin{aligned}
& T_{1}^{2}=\frac{m z_{n}}{A_{A} p_{n}}, \quad 2 \varsigma T_{1}=\frac{c z_{n}}{A_{A} p_{n}}, \\
& \bar{A}_{1}=\frac{A_{1}}{A_{1}}, \quad \bar{A}_{2}=\frac{A_{2}}{A_{A}}, \quad \bar{A}_{e}=\frac{A_{e}}{A_{A}}, \quad \psi_{1}=\frac{p_{1}}{p_{n}}, \quad \psi_{2}=\frac{p_{2}}{p_{n}}, \quad \psi_{e}=\frac{p_{e}}{p_{n}}, \\
& p_{A^{*}}=\frac{\rho \omega^{2}}{2 \pi} \kappa_{1}^{2} A_{A} ; \quad p_{B^{*}}=\frac{\rho \omega^{2}}{2 \pi} \kappa_{2}^{2} A, \\
& K_{1}=\frac{\rho \omega_{n}^{2}}{2 \pi A_{A} p_{n}} A_{A}^{2} \kappa_{1}^{2}, \quad K_{2}=\frac{\rho \omega_{n}^{2}}{2 \pi A_{A} p_{n}} A_{B}^{2} \kappa_{2}^{2}, \quad \Omega=\frac{\omega}{\omega_{n}}, \quad u=\frac{z}{z_{n}} .
\end{aligned}
$$

Equation (2.1) contains an unknown value of the pressure in the annular chamber 9, (Fig.1), which depends on the width of the lateral clearance and can be determined from the equation of continuity of flow through the system of seal clearances: longitudinal 6 and lateral 10. Considering the axial vibrations of the impeller, we omit the inertia of the fluid in these clearances; however, we take into account the expansion and compression of the fluid in chamber 9. For a turbulent flow through the longitudinal 6 and lateral (face) 10 clearances, the continuity equation can be determined as follows (Martsinkovsky and Kundera)

$$
g_{2 n}\left(1+0.19 \varepsilon^{2}\right) \sqrt{p_{1}-p_{B^{*}}-p_{2}}=g_{3 n} u^{1.5} \sqrt{p_{2}-p_{e}}+A_{2} \dot{z}+\frac{V}{E} \dot{p}_{2} .
$$

The first-order differential equation in relation to the $p_{2}$ variable is non-linear. In further transformations, we linearize Eq.(2.5) at static equilibrium, converting it into a variational form

$$
\begin{aligned}
& \frac{V}{E Q_{0}} \delta \dot{p}_{2}+\frac{1}{2}\left(\frac{1}{\Delta p_{20}}+\frac{1}{\Delta p_{c 0}}\right) \delta p_{2}=-\frac{A_{2} z_{n}}{Q_{0}} \delta \dot{u}-\frac{3}{2 u_{0}} \delta u+ \\
& +\frac{1}{2 \Delta p_{20}}\left(\delta p_{1}-\delta p_{B^{*}}\right)+\frac{1}{2 \Delta p_{c 0}} \delta p_{e}+\varepsilon_{c} \delta \varepsilon_{3}
\end{aligned}
$$

where

$$
\Delta p_{20}=\left(p_{1}-p_{B^{*}}-p_{2}\right)_{0}, \quad \Delta p_{c 0}=\left(p_{2}-p_{e}\right)_{0}, \quad \Delta p_{s 0}=\left(p_{1}-p_{B^{*}}-p_{e}\right)_{0} .
$$


Then, dimensionless quantities are introduced into Eq.(2.6), and written in the following linear form (for simplicity, variation signs are omitted)

$$
T_{2} \dot{\psi}_{2}+\psi_{2}=-\kappa_{s}\left(\tau_{2} \dot{u}+u\right)+k_{1} \psi_{1}+k_{2} \psi_{e}+k_{3} \varepsilon-k_{4} \Omega
$$

where

$$
\begin{aligned}
& T_{2}=\frac{2 V p_{n}}{E Q_{0}} \frac{\Delta \psi_{20} \Delta \psi_{c 0}}{\Delta \psi_{s 0}}, \quad \tau_{2}=\frac{2 A_{2} z_{0}}{3 Q_{0}}, \quad \kappa_{s}=\frac{3 \Delta \psi_{20} \Delta \psi_{c 0}}{u_{0} p_{n} \Delta \psi_{s 0}}, \\
& k_{1}=\frac{\Delta \psi_{c 0}}{\Delta \psi_{s 0}}, \quad k_{2}=\frac{\Delta \psi_{20}}{\Delta \psi_{s 0}}, \quad k_{3}=2 \varepsilon_{c} \frac{\Delta \psi_{20} \Delta \psi_{c 0}}{p_{n} \Delta \psi_{s 0}}, \quad k_{4}=2 \frac{A_{A} \Delta \psi_{c 0}}{A_{B} \Delta \psi_{s 0}} K_{2} \Omega_{0} .
\end{aligned}
$$

In the next transformation, we write the linear Eq.(2.8) in the operational form, by substitution $(p \equiv d / d t)$

$$
\begin{aligned}
& \left(T_{2} p+1\right) \psi_{2}=-\kappa_{s}\left(\tau_{2} p+1\right) u+k_{1} \psi_{1}+k_{2} \psi_{e}+k_{3} \varepsilon-k_{4} \Omega, \\
& \psi_{2}=-\kappa_{s} \frac{\tau_{2} p+1}{T_{2} p+1} u+\frac{1}{T_{2} p+1}\left(k_{1} \psi_{1}+k_{2} \psi_{e}+k_{3} \varepsilon-k_{4} \Omega\right) .
\end{aligned}
$$

Relationship (2.11) is substituted into Eq.(2.2), where the last term in the square brackets on the right side is written in a linearized form: $2\left(K_{1}-K_{2}\right) \Omega_{0} \delta \Omega$.

After appropriate transformations, we obtain an equation of the impeller axial vibrations, which takes into account forces generated in the system of seal clearances.

$$
\begin{aligned}
& {\left[\left(T_{1}^{2} p^{2}+2 \varsigma T_{1} p\right)\left(T_{2} p+1\right)+\kappa_{s} \bar{A}_{2}\left(\tau_{2} p+1\right)\right] u=\left[\bar{A}_{2} k_{1}-\bar{A}_{1}\left(T_{2} p+1\right)\right] \psi_{1}+} \\
& +\left[\bar{A}_{2} k_{2}-\bar{A}_{e}\left(T_{2} p+1\right)\right] \psi_{e}+\bar{A}_{2} k_{3} \varepsilon-\left[\bar{A}_{2} k_{4}-2\left(K_{1}-K_{2}\right) \Omega_{0}\left(T_{2} p+1\right)\right] .
\end{aligned}
$$

Arranging the terms according to the order of the differential operator, we obtain the equations of the system in the following form

$$
\begin{aligned}
& \left(a_{0} p^{3}+a_{1} p^{2}+a_{2} p+a_{3}\right) u=-\bar{A}_{1} N_{l}(p) \psi_{1}+ \\
& -\bar{A}_{e} N_{e}(p) \psi_{e}+\bar{A}_{2} k_{3} \varepsilon+2\left(K_{1}-K_{2}\right) \Omega_{0} N_{\omega}(p) \Omega
\end{aligned}
$$

In Eq.(2.13), the operator of unforced vibrations and the operators determining the action of the external forces are as follows

$$
\begin{array}{lc}
D_{0}(p)=a_{0} p^{3}+a_{1} p^{2}+a_{2} p+a_{3}, & N_{1}(p)=T_{2} p+1-k_{1} A_{2} / A_{1}, \\
N_{e}(p)=T_{2} p+1-k_{2} A_{2} / A_{e}, & N_{\omega}(p)=T_{2} p+1-k_{4} \bar{A}_{2} /\left[2\left(K_{1}-K_{2}\right) \Omega_{0}\right] . \\
a_{0}=T_{1}^{2} T_{2}, \quad a_{1}=T_{1}^{2}+2 \varsigma T_{1} T_{2}, \quad a_{2}=2 \varsigma T_{1}+\kappa_{s} \bar{A}_{2} \tau_{2}, \quad a_{3}=\kappa_{s} \bar{A}_{2} .
\end{array}
$$


For the assumed model (in which we omit the inertial hydraulic resistance in the seal clearances), the impeller axial vibrations are described with a third-order differential equation. The operator $D_{0}(p)$ is a natural operator of the system and the equation $D_{0}(p) u=0$ is an equation of the unforced axial vibrations of the impeller.

Further analysis will be limited to the description of the forced vibrations and the system stability, assuming that the variation of the external forces $\psi_{1}, \psi_{e}, \varepsilon$ are described with harmonic functions: $\psi_{1}=\psi_{a l} e^{i \omega t}, \psi_{e}=\psi_{a} e^{i \omega t}, \varepsilon=\varepsilon_{a} e^{i \omega t}$. The responses of the linear system to these excitations (components of the partial solutions) are described using the following formulas

$$
u_{1}=u_{1 a} e^{i\left(\omega t+\phi_{1}\right)}, \quad u_{e}=u_{e a} e^{i\left(\omega t+\phi_{e}\right)}, \quad u_{\varepsilon}=u_{\varepsilon a} e^{i\left(\omega t+\phi_{\varepsilon}\right)} .
$$

Changes in the rotational velocities can be represented as a linear or exponential time function. A response to such changes is determined using time characteristics.

The amplitudes and phases of the harmonic excitations are defined by the frequency transition functions $W(i \omega)$, which are easy to calculate by substituting $p=i \omega$ into the equation of axial vibrations (2.13)

$$
\begin{aligned}
& W_{1}(i \omega)=\frac{u_{1}}{\bar{A}_{1} \psi_{1}}=\frac{u_{l a}}{\bar{A}_{1} \psi_{l a}} e^{i \phi_{1}}=\frac{N_{1}(i \omega)}{D_{0}(i \omega)}, \\
& W_{e}(i \omega)=\frac{u_{e a}}{\bar{A}_{e} \psi_{e a}} e^{i \phi_{e}}=\frac{N_{e}(i \omega)}{D_{0}(i \omega)}, \quad W_{\varepsilon}(i \omega)=\frac{u_{\varepsilon a}}{\bar{A}_{2} \varepsilon_{a}} e^{i \phi_{\varepsilon}}=\frac{k_{3}}{D_{0}(i \omega)} .
\end{aligned}
$$

Isolating the real and imaginary components in the operator of unforced vibrations and in the operators of external forces (2.14), we have

$$
\begin{array}{lll}
D_{0}=U_{0}+i \omega V_{0} ; & U_{0}(\omega)=a_{3}-\omega^{2} a_{2}, & V_{0}(\omega)=a_{2}-\omega^{2} a_{0}, \\
N_{1}=U_{1}+i \omega V_{1}, & U_{1}=1-k_{1} A_{2} / A_{1}, & V_{1}=T_{1}, \\
N_{e}=U_{e}+i \omega V_{e} ; & U_{e}=1-k_{2} A_{2} / A_{e}, & V_{e}=T_{2} ; \quad N_{\varepsilon}=U_{\varepsilon}=k_{3}, \quad V_{\varepsilon}=0 .
\end{array}
$$

Substituting these expressions into formulas (2.17), we obtain frequency transition functions in the form of complex quantities (numbers), for example

$$
W_{l}(i \omega)=\frac{U_{1}+i \omega V_{1}}{U_{0}+i \omega V_{0}}=\frac{u_{1 a}}{\bar{A} \psi_{1 a}} e^{i \phi_{1}}=B_{1}(\omega) e^{i \phi_{I}(\omega)}
$$

where the amplitude- and phase-frequency characteristics describing the action of excitation $\psi_{1}$ have the following form

$$
B_{l}(\omega)=u_{1 a} / \bar{A}_{l} \psi_{1 a}=\left|W_{l}(i \omega)\right|, \quad \phi_{1}(\omega)=\arg W_{l}(i \omega) .
$$


After isolating the real and imaginary parts in the expression $W_{1}$, we have

$$
W_{1}=\frac{U_{1}+i \omega V_{1}}{U_{0}+i \omega V_{0}}=\frac{U_{0} U_{1}+\omega^{2} V_{0} V_{1}}{U_{0}^{2}+\omega^{2} V_{0}^{2}}+i \omega \frac{\left(U_{0} V_{1}-U_{1} V_{0}\right)}{U_{0}^{2}+\omega^{2} V_{0}^{2}}
$$

Finally, the frequency characteristics take the following form

$$
B_{1}(\omega)=\frac{u_{1 a}}{\bar{A}_{1} \psi_{1 a}}=\sqrt{\frac{U_{1}^{2}+\omega^{2} V_{l}^{2}}{U_{0}^{2}+\omega^{2} V_{0}^{2}}}, \quad \phi_{1}(\omega)=\operatorname{arctg} \omega \frac{U_{0} V_{1}-U_{1} V_{0}}{U_{0} U_{1}+\omega^{2} V_{0} V_{1}} .
$$

By analogy, using relationship (2.18), we determine the frequency characteristics taking into account the action of the other excitations

$$
\begin{array}{ll}
B_{e}(\omega)=\frac{u_{e a}}{\bar{A}_{e} \psi_{e a}}=\sqrt{\frac{U_{e}^{2}+\omega^{2} V_{e}^{2}}{U_{0}^{2}+\omega^{2} V_{0}^{2}}}, & \phi_{e}(\omega)=\operatorname{arctg} \omega \frac{U_{0} V_{e}-U_{e} V_{0}}{U_{0} U_{e}+\omega^{2} V_{0} V_{e}}, \\
B_{\varepsilon}(\omega)=\frac{u_{\varepsilon a}}{\bar{A}_{2} \varepsilon_{a}}=\frac{k_{3}}{\sqrt{U_{0}^{2}+\omega^{2} V_{0}^{2}}}, & \phi_{\varepsilon}(\omega)=-\operatorname{arctg} \omega \frac{V_{0}}{U_{0}} .
\end{array}
$$

The frequency characteristics can be used to determine the critical rotational velocities of the impeller at which the vibration amplitudes reach a maximum and the vibration phases change by an angle of $180^{\circ}$.

In the numerical example, the frequency characteristics described with the above relationships were determined for the same data as those used in the calculations of the static characteristics (Kundera and Martsinkovsky).

\subsection{Assessing the stability of axial vibrations}

According to the Routh-Hurwitz stability criterion, the necessary and sufficient condition of stability of a third-order system is that the coefficients of the natural operator (2.14) should satisfy the inequity $a_{1} a_{2}>a_{0} a_{3}$. After the values of the coefficients are substituted into Eqs (2.15), the stability condition is reduced to

$$
(2 \varsigma)^{2}+\left(\frac{T_{1}}{T_{2}}+\kappa_{s} \bar{A}_{2} \frac{T_{2}}{T_{1}} \frac{\tau_{2}}{T_{2}}\right) 2 \varsigma+\kappa_{s} \bar{A}_{2}\left(\frac{\tau_{2}}{T_{2}}-1\right)>0
$$

If the expansion time constant is higher than the compression time constant, $\tau_{2}>T_{2}$, then inequity (2.25) is satisfied at arbitrary values of the damping coefficient $\varsigma$ (also for $\varsigma=0$ ). Using the values of the constants (2.9), we obtain

$$
\frac{A_{2} z_{0}}{3}>\frac{V p_{n}}{E} \frac{\Delta \psi_{20} \Delta \psi_{c 0}}{\Delta \psi_{s 0}}
$$


where: $V=A_{2} H$ - volume of the annular chamber; $H$ - the chamber depth.

Finally, (in the first approximation) the stability condition is reduced to the following inequity

$$
H<\frac{E z_{0}}{3 p_{n}} \cdot \frac{\Delta \psi_{s 0}}{\Delta \psi_{20} \Delta \psi_{c 0}}
$$

The second fraction on the right side of this inequity is defined with formula (2.9). The chamber depth $H$ is an independent parameter, easy to redesign. Thus, the stability condition (2.27) can be used while designing the rotating system of the pump.

\section{Numerical example}

The calculations of dynamic characteristics were performed for an impeller of a modernized high speed single-stage centrifugal pump. The pump modernization involved applying an impeller with a system of seal clearances, shown in Fig. 1.

The static calculations for the modernized pump are omitted here because they are presented in the previous work of the authors (Kundera and Martsinkovsky).

1) Input parameters.

Let us assume the following geometrical dimensions of the pump impeller

$$
\begin{aligned}
& R_{1}=56 \mathrm{~mm}, \quad R_{2}=75 \mathrm{~mm}, \quad R_{3}=65 \mathrm{~mm}, \quad R_{4}=58 \mathrm{~mm}, \quad R_{5}=51 \mathrm{~mm}, \quad R_{6}=15 \mathrm{~mm}, \\
& l_{1}=l_{2}=15 \mathrm{~mm}, \quad H_{1}=H_{2}=0.2 \mathrm{~mm} \text { (with symbols being the same as those in Fig. 1). }
\end{aligned}
$$

The assumptions concerning the pump performance parameters are

$$
\begin{aligned}
& p_{n}=3.5 \mathrm{MPa} ; \quad p_{e}=1.25 \mathrm{MPa} ; \quad \omega_{n}=1500 \mathrm{~s}^{-1} ; \\
& \rho=10^{3} \mathrm{~kg} / \mathrm{m}^{3} ; \quad \kappa_{1}=0.8 ; \quad \kappa_{2}=0.6 .
\end{aligned}
$$

2) Calculation of the surface areas of the impeller

$$
\begin{aligned}
& A_{A}=\pi\left(R_{2}^{2}-R_{1}^{2}\right)=7.58 \cdot 10^{-3} \mathrm{~m}^{2}, \quad A_{B}=\pi\left(R_{2}^{2}-R_{3}^{2}\right)=4.08 \cdot 10^{-3} \mathrm{~m}^{2}, \\
& A_{2}^{\prime}=\pi\left(R_{3}^{2}-R_{4}^{2}\right)=2.76 \cdot 10^{-3} \mathrm{~m}^{2}, \quad A_{c}=\pi\left(R_{4}^{2}-R_{5}^{2}\right)=2.40 \cdot 10^{-3} \mathrm{~m}^{2}, \\
& A_{2}=A_{2}^{\prime}+0.5 A_{c}=3.96 \cdot 10^{-3} \mathrm{~m}^{2}, \quad A_{e}^{\prime}=\pi\left(R_{1}^{2}-R_{5}^{2}\right)=1.68 \cdot 10^{-3} \mathrm{~m}^{2}, \\
& A_{3}=\pi R_{6}^{2}=7.07 \cdot 10^{-4} \mathrm{~m}^{2}, \quad A_{e}=A_{e}^{\prime}+A_{3}-0.5, \quad A_{c}=1.19 \cdot 10^{-3} \mathrm{~m}^{2}, \\
& \bar{A}_{1}=\left(A_{A}-A_{B}\right) / A_{A}=0.437 ; \quad \bar{A}_{2}=A_{2} / A_{A}=0.45 ; \quad \bar{A}_{e}=A_{e} / A_{A}=0.152 .
\end{aligned}
$$


Coefficients (4): $K_{1}=0.312 ; \quad K_{2}=0.056$.

3) Calculation of the frequency characteristics for the impeller-seal clearances system.

To calculate the dynamic characteristics, we had to assume the following additional parameters: $m=30 \mathrm{~kg}, E=2 \cdot 10^{9} \mathrm{~Pa}, \mu=10^{-3} \mathrm{~Pa} \cdot \mathrm{s}, \varsigma=0.05, \varepsilon_{c}=0$.
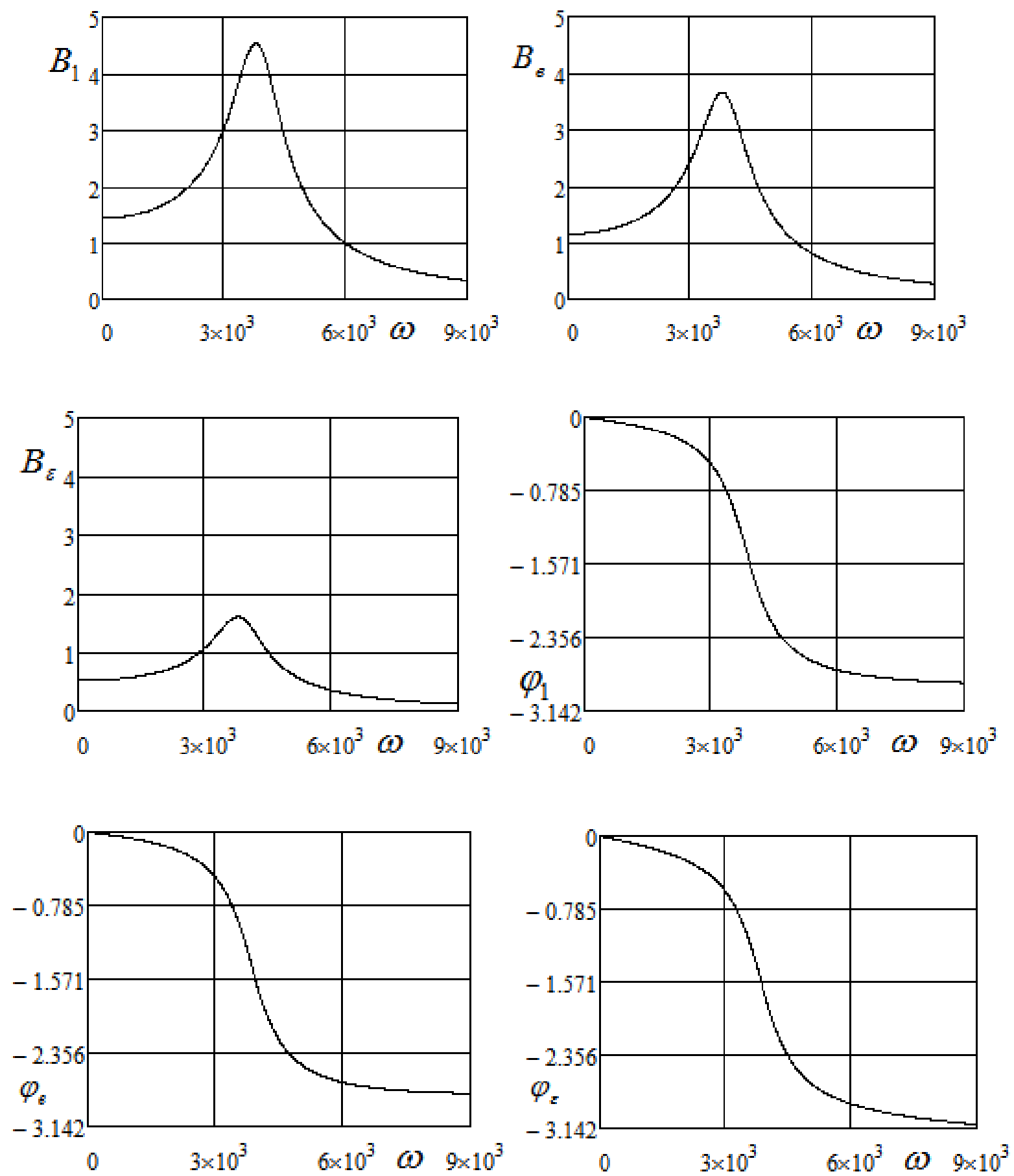

Fig.2. Amplitude vs. frequency and phase vs. frequency characteristics. 
From the frequency characteristics it is evident that the first resonance occurs at frequencies of $3.79 \times 10^{3} \mathrm{~s}^{-1}$. The second natural frequency of the system axial vibrations exceeds the ranges specified for the admissible impeller rotational velocities.

Relationships (2.22)-(2.24) can be used to calculate the values of the resonance vibration amplitudes. If we assume relative values of the amplitudes of the external forces: $\psi_{l a}=\psi_{e a}=\varepsilon_{a}=0.1$, then the impeller axial vibration amplitudes are: $z_{l a} \approx 0.21 z_{n}=0.058 \mathrm{~mm}, \quad z_{e a} \approx 0.06 z_{n}=0.017 \mathrm{~mm} ; \quad z_{\varepsilon a} \approx 0.024 z_{n}=$ $=0.007 \mathrm{~mm}$, respectively.

The results indicate that the critical angular (rotational) velocity is twice as large as the assumed pumping velocity. This confirms that the impeller is rigid because of the axial vibrations. Furthermore, the resonance amplitudes corresponding to the critical rotational velocity are not dangerous.

\section{Conclusions}

In the first approximation, the impeller with a balancing device performs independent axial vibrations, which is a result of the kinematic excitation in the form of predetermined radial vibrations caused by static unbalance.

With the axial vibrations of the impeller, variable throttling of the fluid through the lateral (face) clearance acts as negative feedback. Thus, the impeller with a balancing device (containing two longitudinal and one lateral seal clearances) becomes a system of automatic control of the axial position of the impeller. Knowing the dynamic rigidity of the controller, we can calculate approximate values of natural frequencies and assess the stability of the axial vibrations. More accurate values of the natural frequencies are used to plot amplitude and phase characteristics. The analysis of the dynamics of the impeller with a balancing device showed that the rotating system of the pump is characterized by stability for a wide range of rotational velocities.

Observations of the prototypes of centrifugal pumps modernized in this way have been conducted both under laboratory and factory conditions. The findings, which confirm their numerous advantages over conventional pumps, include:

- better vibroacoustic characteristics;

- higher reliability and a longer service life between overhauls;

- easier operation, assembly and transport. the drive shaft.

It is also possible to reduce the weight and size of such pumps by eliminating the external bearing of

\section{Nomenclature}

$$
\begin{aligned}
A_{A}, A_{B} & - \text { outer surfaces area of the impeller shrouds }\left[\mathrm{m}^{2}\right] \\
\bar{A}_{1}, \bar{A}_{2}, \bar{A}_{e} & - \text { dimensionless surface areas Eq.(2.4) } \\
a_{o}, a_{1}, a_{2}, a_{3} & - \text { parameters in Eq.(2.13) } \\
B_{I}(\omega), B_{e}(\omega), B_{\varepsilon}(\omega) & - \text { amplitude frequency characteristics } \\
D_{o}(p) & - \text { natural operator of the system } \\
E & - \text { compression modulus }\left[\mathrm{N} / \mathrm{m}^{2}\right] \\
e & - \text { radial displacement of the impeller axis } \\
g_{2 n}, g_{3 n} & - \text { coefficients capacities of the clearances Eq. }(2.5) \\
H & - \text { the annular chamber depth }[\mathrm{m}] \\
H_{1}, H_{2} & - \text { width of the longitudinal clearances }[\mathrm{m}]
\end{aligned}
$$




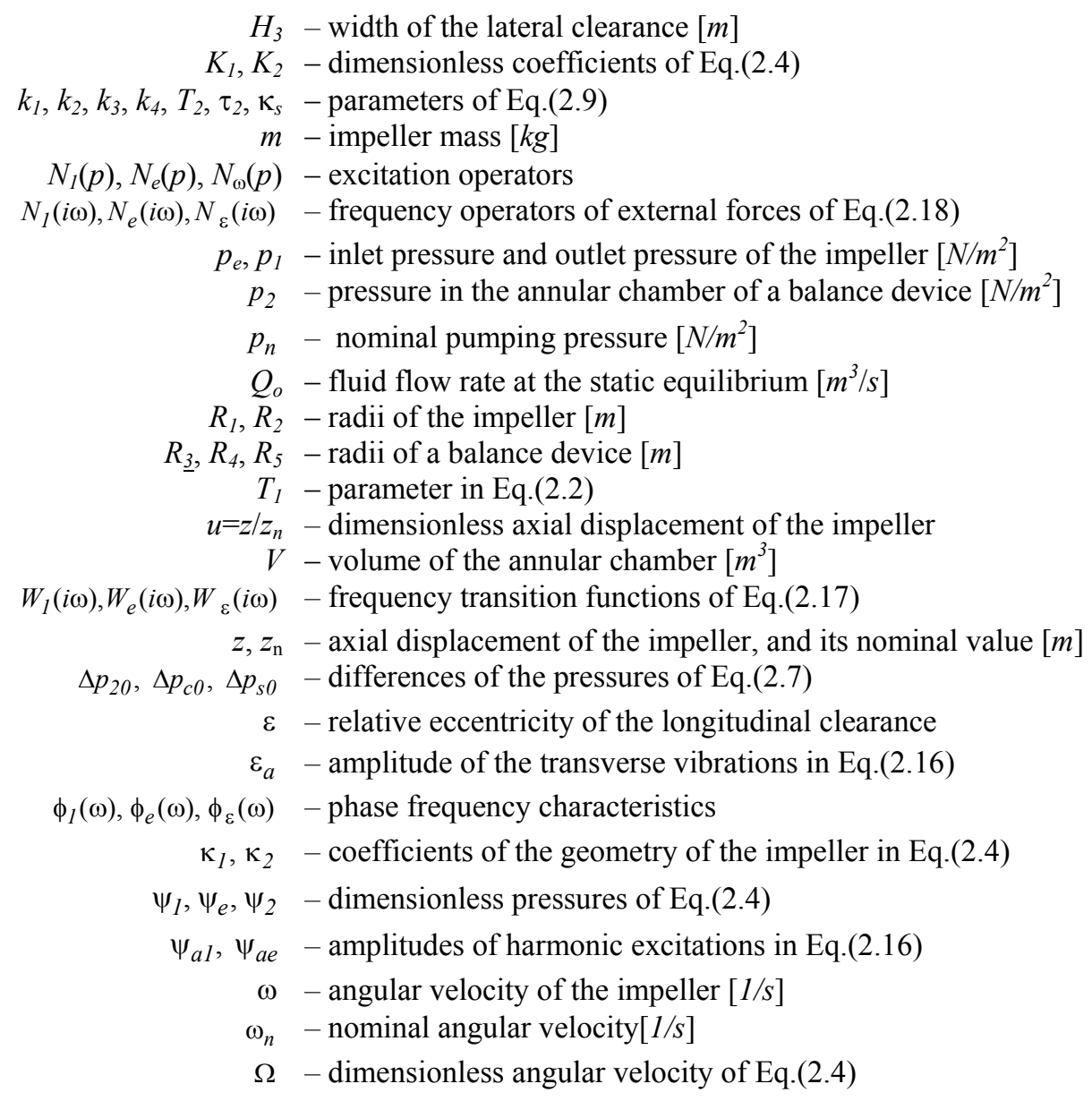

\section{References}

Antoszewski B. and Rokicki J. (2006): Influence of heterogeneous surface on fluid flow in mechanical seals. Chemical and Process Engineering, vol.27, No.3, pp.889 - 899.

Childs D.W. (1993): Turbomachinery Rotordynamics Phenomena, Modeling, and Analysis. - John Wiley and Sons, Inc.

Faria M.T.C. and Miranda W.M. (2012): Pressure dam influence on the performance of gas face seals. - Tribology International, No.47, pp.134-141.

Gosiewski Z. (2008): Analysis of coupling mechanism in lateral/torsional rotor vibrations. - Journal of Theoretical and Applied Mechanics, vol.46, No.4, pp.829-844.

Jędral W. (2001): Centrifugal Pumps. - Warsaw: PWN. (in Polish)

Kalinicienko P.M. and Suprun A.B. (2011): Effective ways of balancing the centrifugal pump impeller. - Monograph Vibration Reliability and Hermetic Sealing Centrifugal Machines, Edited by Martsinkovsky V.A. and Zagorulko A.W., Sumy, pp.243-249. (in Russian)

Korczak A. (2005): Investigations of systems counterbalancing the arial thrust in multi-stage cetrifugal pumps. Scientific Book of the Silesian University of Technology, No.1679, Gliwice. (in Polish). 
Kundera C. and Martsinkovsky V.A. (2014): Static and dynamic analysis of a pump impeller with a balancing device. Part I: Static analysis. - Int. J. of Applied Mechanics and Engineering, vol.19, No.3, pp.609-619.

Li W., Yang Y., Sheng D. and Chen J. (2011): A novel nonlinear model of rotor/bearing/seal system and numerical analysis. - Mechanism and Machine Theory, No.46, pp.618-631.

Martsinkovsky W. and Kundera C. (2008): Design theory of noncontacting seals. - Editorial of the Kielce University of Technology, Kielce. (in Polish)

Received: May 15, 2014

Revised: May 16, 2014 